\title{
Through-Thickness Velocity Profile Measurements in an Elastohydrodynamic Contact
}

Aleks Ponjavic, Mourad Chennaoui, Janet S. S. Wong

Department of Mechanical Engineering

Imperial College London

London, SW7 2AZ

Janet S. S. Wong

j.wong@imperial.ac.uk

Department of Mechanical Engineering

Imperial College London

London, SW7 2AZ

02075948991

This work presents for the first time through-thickness velocity profiles obtained in an EHL contact by photobleached imaging. The velocity profile was inferred by following the evolution of the shape of a photobleached plug formed through the thickness of the fluorescently-doped lubricant, oligomer polybutene $(\mathrm{PB})$, in the contact when shear was applied. The proposed methodology was validated by successfully obtaining the expected linear profile with PB experiencing Couette flow. The methodology was then applied to PB in an EHL contact. The variation of the profiles within the contact area was also investigated.

The velocity profile of PB in an EHL contact severely deviates from the common linear assumption and exhibits inhomogeneous shear: three regions of varying shear rate have been observed. The phenomenon is shown to be neither due to thermal nor diffusion effects. PB also shows significant slip at the glass-liquid interface. The amount of slip varies with position in the contact. Possible causes, such as pressure induced viscosity enhancement, as well as the significance of the findings and the benefits of the technique are discussed.

The linear velocity profile in an EHL contact is usually assumed for both the film thickness and friction predictions. The profile has however never been measured experimentally until now. This work enables the validation of conventional assumptions and the study of flow heterogeneity of lubricants in a contact. This facilitates an improved understanding of the rheology of confined lubricant and hence more accurate predictions of tribological properties.

EHL, velocity profile, lubricant flow, fluorescence, interfacial slip, thin film keywords 4-6

EHL:Elastohydrodynamic lubrication;PB:Polybutene 
Lubricants are frequently used in engineering systems to improve efficiency by manipulating their tribological performance. Operating conditions, including viscosity and film thickness, are chosen such that wear rate is minimized and friction can be controlled. The system operates in the elastohydrodynamic lubrication (EHL) regime when the normal stress applied to the contact is high enough such that elastic deformation of the rubbing surfaces occurs. The lubricant film thickness is typically in the sub-micrometer range and predictions of film thickness and friction are crucial for components designed to operate in the EHL regime. Numerical and theoretical expressions used for such purposes have been developed, with varying degrees of success. The derivation of these expressions requires the knowledge of the velocity profile of the fluid [1]. An EHL contact formed by two surfaces sliding relative to each other is shown in Fig. 1, together with the most commonly assumed velocity profile of the lubricant. This profile can be described using the Reynolds equation where, assuming the pressure gradient is small, the velocity of the lubricant should vary linearly through the thickness of the film [2]. It should be noted that if the assumed velocity profile is inappropriate, the predictions made based on this profile will be inaccurate. In this work, the goal is to develop a methodology capable of obtaining the through-thickness profile in an EHL contact. It will then be used to verify the universality of the linear velocity profile in a low speed, sliding tribological contact.

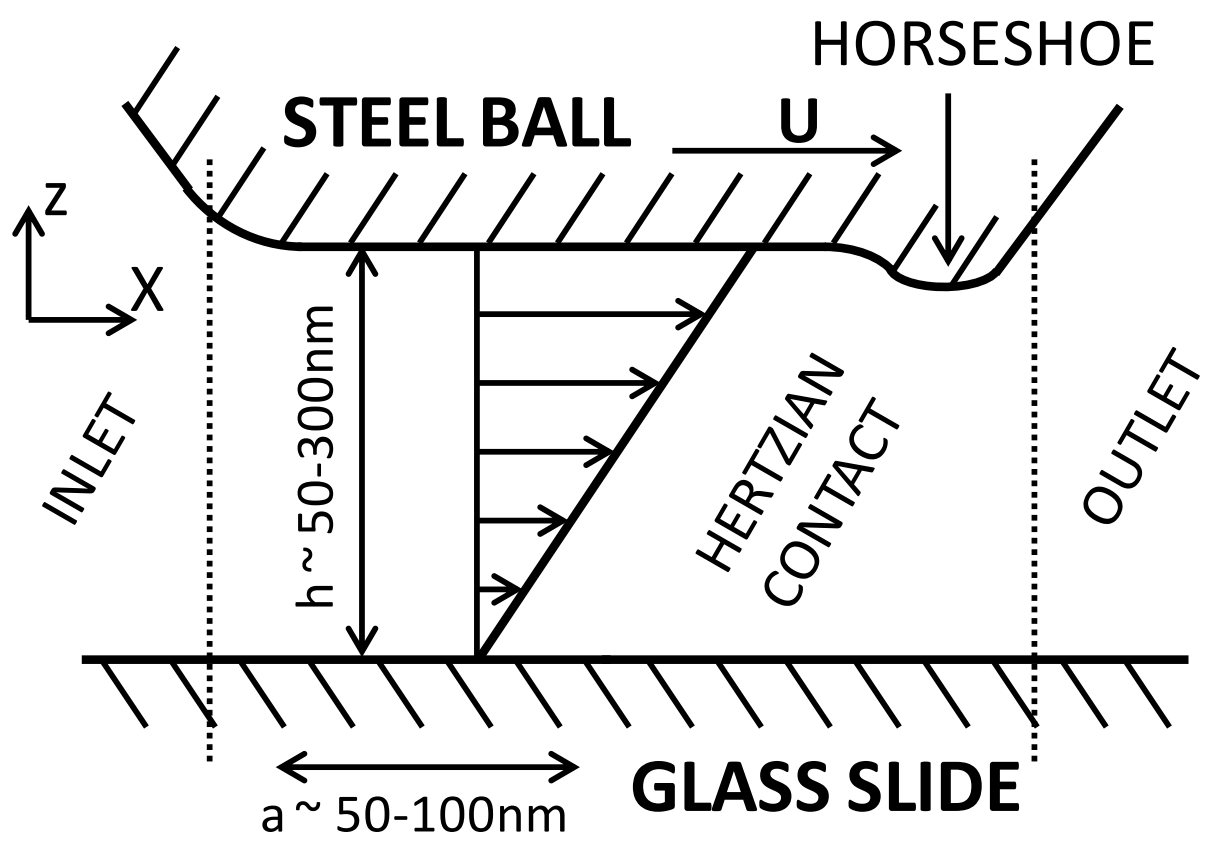

Fig. 1 Schematic of an EHL contact. The surface of the steel ball is moving at a velocity $U$. A lubricant film with mostly constant sub-micron thickness is created in the Hertzian contact. A linear (Couette) velocity profile is indicated by arrows. A diminution of the film thickness in the outlet is associated with a pressure spike.

The relatively high shear rate and pressure in an EHL contact cause severe changes in the rheology of the lubricant [3]. It can become viscoelastic, and in some cases it may even display glassy behaviour [4]. It has been shown that lubricants can solidify $[5,6]$ at pressures commonly encountered in EHL applications. These observations suggest that the commonly assumed linear velocity profile may be inappropriate. Alternative models describing the velocity profile have been suggested. Ehret et al. [7] assumed that the lubricant was liquid near the two bounding surfaces, but that in the center of the film, it 
flowed like a solid plug. This has been used to successfully explain some anomalous behaviour seen in EHL with high viscosity lubricants. Meurisse and Espejel [8] argued that there is adhesion between the lubricant and the surfaces at molecular scales. This caused them to develop a theoretical framework, where the lubricant is separated into layers of varying viscosity. The phenomenon is commonly referred to as inhomogeneous shear and it describes a significant localized variation in rheology accompanied by a change in the local shear rate and hence the velocity profile. Such changes could be caused by localized changes in viscosity which in turn could be induced by heating, pressure, shear or confinement. Inhomogeneous shear has been seen in shear flows of foams [9], entangled polymers [10] and yield stress fluids [11]. There are two types of inhomogeneous shear. Thermally induced shear inhomogeneity can appear when there is a mismatch between the heat transfer rates at the two sliding surfaces. A temperature gradient is then induced in the liquid, which causes a variation in viscosity and therefore affects the velocity profile. Mechanically induced shear inhomogeneity, which can be highly localised, occurs when lubricant solidifies and effectively yields under the applied stresses. Bair et al. have developed a high pressure rheometer [12], capable of measuring the rheology of lubricants at conditions comparable to those encountered in EHL. Using particle image velocimetry, they showed that pressure can induce mechanical inhomogeneous shear, although the film thickness studied was much larger than that in an EHL contact. Molecular dynamics has been used to further probe the nature of pressurised shear flows. Heyes et al. [13] studied a pressurised simple Leonard-Jones liquid in plane Couette flow. They showed that the liquid does indeed show significant inhomogeneous shear when conditions similar to EHL are applied. Furthermore the shear inhomogeneity is not always restricted to the surfaces, but in some cases it occurs at the center of the film.

Apart from the existence of inhomogeneous shear, Heyes et al. [13] also showed that the particular liquid simulated can slip at the wall. It is well known that polymers [14] and nonwetting liquids [15] slip when subject to a sufficient shear stress. Given the high stresses in an EHL contact, slip is quite likely. Choo et al. [16] showed that a large reduction in friction was observed when a glass surface was treated with a hydrophobic coating. They explained this reduction by claiming that the lubricant must slip at the liquid-solid interface. Since friction was the only measurement made, no comment on the variation of slip within the contact could be made, and an average property was assumed. Guo et al. [17] estimated the slip length of polybutene (PB) in an EHL point contact. A dimple in the film thickness was created by instantaneous loading of the contact. By assuming a linear velocity profile, the motion of the dimple through the contact could be related to the slip length. Again, the slip length found was inferred and was assumed not to vary with position in the contact.

The velocity profile in a lubricated tribological contact has not yet been measured experimentally. An experimental scheme that allows the continuous mapping of fluid velocities in the thickness direction is necessary to obtain such information. This is challenging, especially in EHL where films commonly have submicron thickness. The use of velocimetry at scales below the diffraction limit is a difficult task. Nevertheless, some contemporary techniques are capable of performing such measurements. Stimulated emission depletion, combined with photobleaching anemometry [18], has been used to determine the velocity of pressure-driven flow of water through a nanocapillary. The measurements were done in steps of $35 \mathrm{~nm}$ through the film thickness. Thus only a few measurements could be performed through the thickness of a $100 \mathrm{~nm}$ fluid film typical to EHL using this approach. Nano-particle image velocimetry techniques $[19,20]$ have been implemented yielding through-thickness resolutions of about $50 \mathrm{~nm}$, also making them inappropriate for use in EHL velocity profile determination. The necessary size of nanoparticles used $(\sim 20 \mathrm{~nm})$ is also prohibitively large due to particle-wall interactions 
[21]. Smaller probes, such as single molecules, could potentially be used to overcome this problem. However, they also face the challenge of the diffraction limit and offer similar thickness-resolution limitations as micro- and nano-particle image velocimetry with a significant reduction in the signal to noise ratio. Atomic force microscopy [22] can achieve a higher spatial resolution, but its application in an EHL contact is difficult due to access limitations. Fluorescence imaging has been used to study the flow in a contact under rolling conditions [23]. In [23], fluorescently doped lubricant was added at the inlet of the contact which was originally flooded with undoped lubricant. The motion of doped lubricant was monitored as it moved across the contact. It was shown that the pressuredriven flow is negligible and that there is an increase in relative velocity close to the location with minimum film thickness. While this result is unique, as no other direct velocity measurement for an EHL contact exists, it is an average velocity and reveals no information about the variation of velocity through the film thickness. Therefore, the technique would not be appropriate for a sliding contact.

Photobleached imaging has been used to study slip [24-26], as well as diffusion in electro-osmotic flows [27]. The technique shows many similarities to caged-fluorescence imaging $[28,29]$, which is part of the greater field of molecular tagging velocimetry. Most recently, photobleaching has been used to measure dispersion and diffusion coefficients in submicron geometries [30]. Using an extended analysis, it has also been used to reconstruct the velocity profile in a gravity driven thick film with Poiseuille flow [31]. Ultimately, the geometry independence and the velocity reconstruction capability make the technique a suitable candidate for velocimetry in an EHL contact.

In this paper, photobleached imaging is applied to determine the through-thickness velocity profile in an EHL contact. A fluorescent dye is dissolved in a test lubricant and a high intensity focussed laser is used to bleach a small column of fluid (photobleached plug) within the lubricated, sliding contact. The movement and shape of this photobleached plug is then monitored by using a low intensity laser to map the fluorescence intensity of the lubricant film over time.

The aim of this paper is to demonstrate the ability of the proposed methodology to measure the through-thickness velocity profile in a thin film, and to successfully apply the technique to an EHL contact. This work focuses on obtaining the resolution of the methodology and its sensitivity in detecting potential behavioural heterogeneities in the contact. The effects of load and shear rate on the obtained results, while important, are a topic for further study. First, the experimental setup is described. Then, the theoretical framework used for analysing the data and reconstructing the velocity profile is defined. A submicron plane Couette flow was used to validate the framework, and to show that the correct velocity profile can be reconstructed. The technique was then applied to an EHL point contact, showing that the velocity profile severely deviates from theoretical predictions. The direction of the two-dimensional flow within the contact was determined, showing how the liquid flowed around the contact, and how it was squeezed out due to the pressure gradient. Finally, measurements were made at various positions in the contact, where the slip length was extracted and compared to the pressure distribution.

\section{Experimental}

Through-thickness velocity profiles of a lubricant were obtained for two flow conditions: a plane Couette flow created by shearing a thin film of liquid between parallel glass slides; and an EHL point contact created by squeezing the lubricant between a rotating steel ball and a stationary glass slide. The confined lubricant was doped with fluorescent dye and was placed on top of an inverted microscope. Photobleached imaging was used to tag a volume of the fluid. Observing and analysing the evolution of the shape of this tagged 


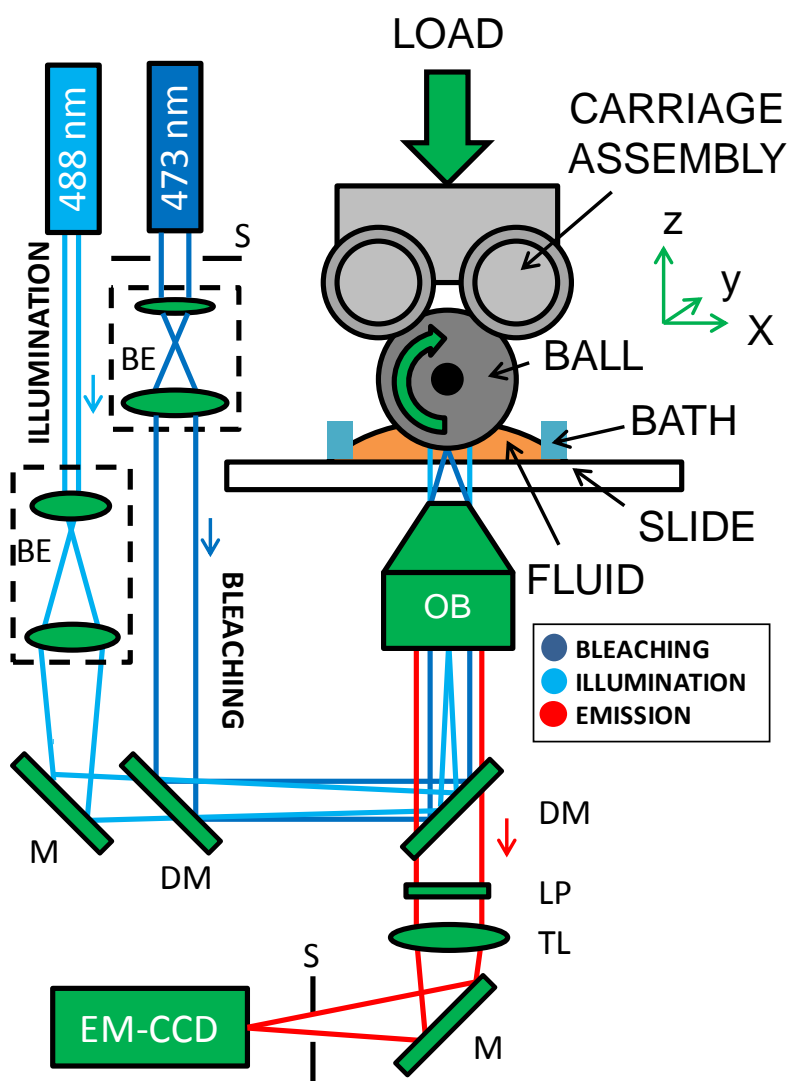

Fig. 2 Schematic of the photobleached imaging setup. BE: Beam expander, S: Shutter, M: Mirror, DM: Dichroic mirror, OB: Objective, LP: Long pass filter, TL: Tube lens, EM-CCD: Electron multiplying charge coupled device. The EHL contact setup is shown in the figure, which can be replaced with parallel plate glass slides for plane Couette flow (not shown).

A schematic of the experimental setup is presented in Fig. 2. The sample was either a film suspended between two glass slides (not shown), or a film created using a loaded rotating ball (shown in Fig. 2). A Zeiss Axiovert 200M inverted microscope with a 10x (0.25 NA), 20x (0.4 NA) or 50x (0.7 NA) objective was used for imaging. An Andor Ixon3 860 electron multiplying charged coupled device camera was used for acquisition. Shutter heads and a shutter control box by Stanford Research Systems controlled the bleach duration and protected the camera during bleaching.

Blue (473 nm at $20 \mathrm{~mW}$ on stage) and cyan ( $488 \mathrm{~nm}$ at $12 \mathrm{~mW}$ on stage) solid state lasers, operating in TEM00 mode, were used in this experiment for creating the tagged volume for flow profile reconstruction through photobleaching and illuminating the observation volume by laser excitation respectively. The power experienced by the sample was around $40 \mathrm{~mW}$ and $24 \mathrm{~mW}$ from the blue and cyan lasers respectively due to reflection at the steel surface. The cyan illumination beam was expanded and focused onto the back of the objective resulting in epi-fluorescence illumination. Fluorescent probes were dissolved into model lubricant. When illuminated by the cyan laser, the lubricant emitted homogenous fluorescence. The blue laser was used as a bleaching beam to create a small photobleached plug, in the form of a dark cylinder within the fluorescent probe volume, through the thickness of the confined lubricant. This plug was 
the tagged volume, whose evolution in shape was followed as the model lubricant was sheared. The plug had an inverse Gaussian intensity distribution in x- and y-directions, and a homogenous intensity distribution in z-direction. The blue laser passed through a shutter which controlled the time the lubricant was exposed to the bleach beam, and hence the diameter of the plug. After the blue laser was expanded and collimated using a beam expander, it rejoined the cyan laser through a dichroic beam splitter and was focused onto the sample stage. The fluorescence emission from the sample was collected through the objective and the dichroic mirror. It then passed through a long pass filter to remove the residual signal from the cyan laser. Finally, a tube lens focused the emission onto the chip of the camera. A shutter was installed just in front of the camera to protect it from the bleaching beam. For the 20x objective, the radius of the diffraction limited volume was $3.60 \mu \mathrm{m}$, and the depth of field was $1.52 \mu \mathrm{m}$. For the $50 x$ objective, the radius of the diffraction limited volume was $1.50 \mu \mathrm{m}$, and the depth of field was 480 $\mathrm{nm}$. The film thickness was smaller than the depth of field of the objective for the experimental conditions used in this work, ensuring that a through-thickness photobleached plug was generated during this process.
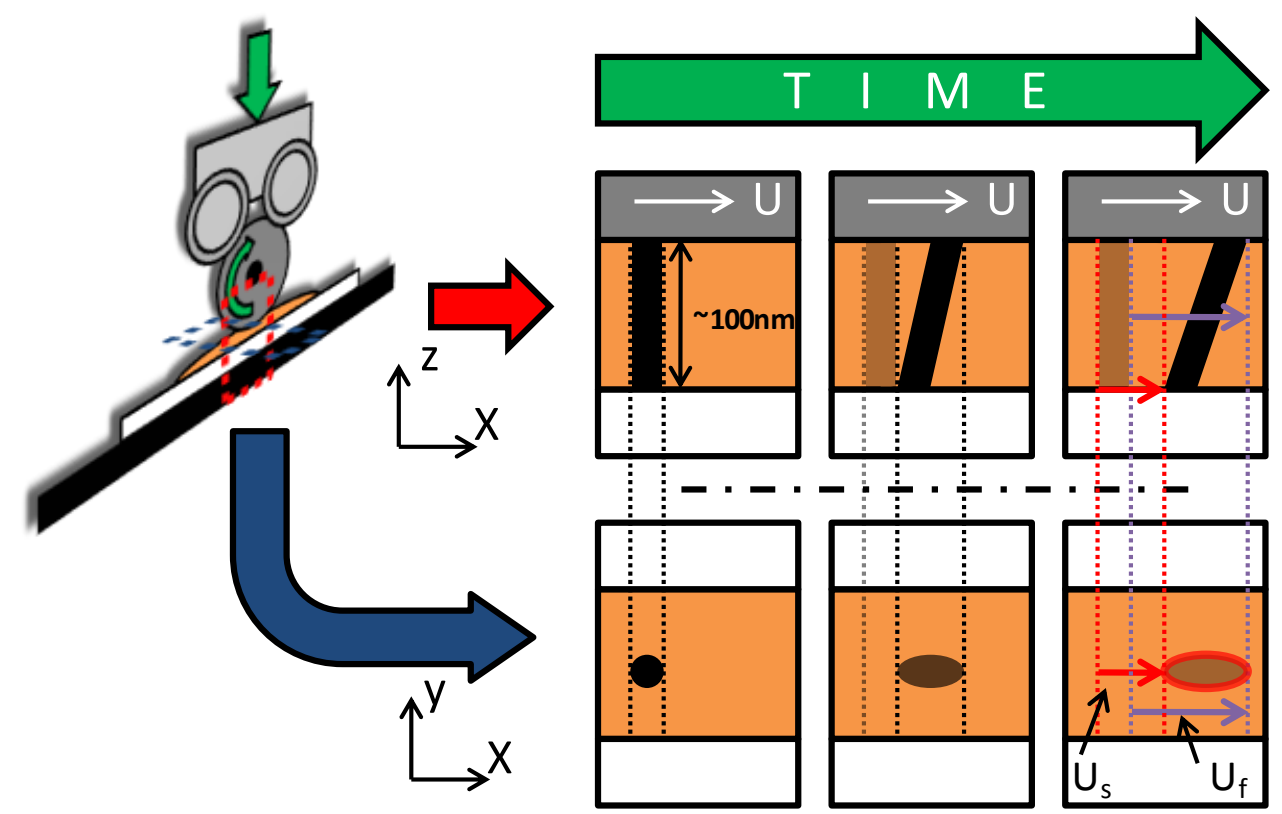

Fig. 3 Schematic of the photobleached imaging setup with a sketch of the spatiotemporal evolution of the photobleached plug. $U_{s}$ is the slip velocity and $U_{f}$ is the velocity of the front of the plug.

\subsection{Photobleached imaging for flow profiling}

Fig. 3 shows a schematic of the spatiotemporal response of a photobleached plug, in a fluorescently doped lubricant, due to Couette flow in the x-direction, with interfacial slip (slip velocity of $U_{s}$ ) at the bottom interface. The photobleached plug has much lower fluorescence intensity than its surrounding fluorescent lubricant. The $x-z$ plane depicts the through-thickness view of the confined lubricant, which cannot be visualised experimentally due to the submicron thickness of the film. The $x-y$ plane corresponds to the image plane, which was observed in the experiment. The intensity distribution within the photobleached plug is initially Gaussian, as it is produced by a diffraction-limited focused laser beam. The convection of the fluid causes the shape of the plug to change with time. In the case presented in Fig. $\mathbf{3}$, the observed plug elongates in the $\mathrm{x}$-direction 
and the back of the spot moves with a velocity $U_{s}$. Other velocity profiles due to Poiseuille flow and inhomogeneous shear would produce distinctly different results where the intensity distribution in $x$ becomes distorted. Both the elongation of the plug and the interfacial velocity of the lubricant can be measured experimentally. The evolution of the spot can be used to reconstruct the unknown through-thickness velocity profile.

\subsection{Acquisition}

Acquisition of images was achieved using a Labjack U6 digital acquisition system. Four background images were taken prior to bleaching. The shutter controlling the bleaching beam was then opened for a set duration. Subsequently, 16 frames were acquired at a given exposure time, capturing the response of the bleached plug. Synchronous averaging was employed to reduce noise [32]. Due to the high viscosity and the small film thickness of the model lubricant used in this study, the characteristic time for through thickness flow profile development is of the order of $10^{-16}$ second. Thus steady state conditions required for synchronous averaging can be assumed. While the flow is developed, it is possible that there are transient changes in the microstructure of the liquid. Such changes would not be resolved by the technique but an average result would still be acquired. A delay between each sequential acquisition was employed to allow most of the photobleached dye to exit the observation volume. The camera background $I_{b}$ was removed from the image sequences and the final result was the normalised photobleached intensity $I_{N}$ given by $I_{N}(t)=\left(I_{0}-I(t)\right) / I_{0}$ where $I_{0}$ is the pre-bleach intensity and $I(t)$ is the intensity at time $t$.

\subsection{Fluid and tracer}

The fluid investigated in this work was PB H-300 (supplied by Ineos Oligomers). PB is an oligomer which has high viscosity at relatively low molecular weight. With a number average molecular weight of $1300 \mathrm{~g} / \mathrm{mol}, \mathrm{PB} \mathrm{H}-300$ has a low shear rate viscosity, $\mu_{0}$, of $100 \mathrm{~Pa} \mathrm{~s}$ at $20^{\circ} \mathrm{C}$ and $0.62 \mathrm{~Pa} \mathrm{~s}$ at $100^{\circ} \mathrm{C}$ (measured using a TA AR2000Ex rheometer). A fluorescent probe, nile red (Sigma-Aldrich), was dissolved into the PB at a concentration of $1 \mathrm{mM}$ by magnetic stirring at $150^{\circ} \mathrm{C}$. No surface adsorption of the dye to either glass or steel could be seen during the experiments.

PB is commonly considered to be a Newtonian fluid, but at higher molecular weights, and more importantly, at higher pressures, it is known to shear thin at the shear rates encountered in this work [3]. Here, a peak pressure, $p$, of approximately $0.315 \mathrm{GPa}$ is applied when PB is confined in an EHL contact. Using the Barus equation $\left(\mu=\mu_{0} e^{\alpha p}\right.$, where $\alpha=32 \mathrm{GPa}^{-1}$ is the pressure viscosity coefficient measured using interferometry), the viscosity, $\mu$, of $\mathrm{PB} \mathrm{H}-300$ at such pressure is about $2.4 * 10^{6} \mathrm{~Pa}$ s. With a glass viscosity, $\mu_{g}$, of $10^{7} \mathrm{~Pa} \mathrm{~S}$ [3], PB can be very viscous, or even partially or completely solidify under the experimental EHL conditions. While the pressure applied in this work may not cause common lubricants such as mineral oil ( $\mu_{g}=10^{7} \mathrm{Pas}, \alpha=23.1 \mathrm{GPa}^{-1}$ ) [33] and polyalphaolefin $\left(\mu_{g}=10^{6} \mathrm{Pas}, \alpha=12.6 \mathrm{GPa}^{-1}\right)$ [33] to solidify, pressures in rolling element bearings readily reach up to $4 \mathrm{GPa}$, at which point enhanced viscosity has been observed $[5,6]$. Hence the behaviour observed with $\mathrm{PB}$ in this work is relevant to common lubricants. The potential of PB to deviate from commonly assumed flow behaviour in the applied conditions allows the effectiveness of the proposed methodology to be demonstrated: if PB displays a non-linear velocity profile, the detection of such a profile would show that the proposed methodology is appropriate.

Nile red is usually considered to be a photostable fluorophore [34]. When dissolved in PB, a relatively fast photobleaching rate constant of $20 \mathrm{~ms}$ was achieved using $473 \mathrm{~nm}$ 
excitation (40 $\mathrm{mW}$ on stage) with a $50 x$ objective. A typical bleach time of $10 \mathrm{~ms}$ resulted in a $40 \%$ peak reduction in intensity for the PB-nile red solution. Heating during photobleaching needs to be considered as it could cause a change in the viscosity of the fluid [35]. Finite element simulations of heat transfer were performed for an axisymmetric Gaussian heat source in PB using Elmer (http://www.csc.fi/english/pages/elmer). An analytical heat source equivalent to the heating experienced by PB due to photobleaching under the experimental conditions was generated based on an infinite cylindrical Gaussian heat source surrounded by PB (unbound case) using the approach outlined in [36]. The simulated temperature rise in the unbound case agreed well with the analytical solution [36]. A simulation was then performed using the experimental EHL conditions, with PB bounded by steel and glass (bounded case). The resultant steady state temperature rise was $3 \mathrm{~K}$. This would cause an approximate change in viscosity of $5 \%$. The temperature gradient would however decay within $1 \mathrm{~ms}$ after photobleaching. Hence over the experimental time scale (about $100 \mathrm{~ms}$ ), the viscosity change due to photobleaching would be negligible. Control experiments were conducted with various combinations of nile red concentrations and laser bleaching powers to lower the amount of heat experienced by PB. No significant change in the determined velocity profiles was observed, confirming that the results obtained are not affected by heating due to photobleaching.

The determination of the through-thickness velocity profile by the observation of the change in the shape of the photobleached plug with time requires the Brownian diffusion coefficient of the fluorescent probe, $D$, in the contact to be quantified. Due to the squeeze film effect, $D$ is not a trivial quantity to measure in a tribological contact. A submicron film of PB H-300 was prepared between two glass slides. Fluorescence recovery after photobleaching [37] was used to measure $D$ in the PB film. It was found to be $3.8 \times 10^{-15} \pm$ $1.1 \times 10^{-15} \mathrm{~m}^{2} / \mathrm{s}$. This value can be compared to the prediction from the two-dimensional Stokes-Einstein diffusion equation $D=k_{b} T /\left(4 \pi \mu_{0} r\right)$ where $k_{b}$ is the Boltzmann constant, $T$ is the temperature and $r$ is the hydrodynamic radius. At a temperature of $20^{\circ} \mathrm{C}$, a kinematic viscosity of $100 \mathrm{~Pa} \mathrm{~s}$ and a radius of $0.5 \mathrm{~nm}$ [38], the theoretical diffusion coefficient of nile red in PB H-300 is $6.4 \times 10^{-15} \mathrm{~m}^{2} / \mathrm{s}$, which is comparable to the experimental value. The measured $D$ is the upper bound value for the diffusion of nile red in PB. In an EHL contact, $D$ would be significantly smaller due to the pressure experienced by PB. The effect of dye diffusion on data interpretation will be discussed in the next section.

\section{Analysis}

\subsection{Experimental framework}

The observation volume was homogeneously illuminated. The photobleaching of the dye 38 by the bleaching beam in the contact caused the appearance of a photobleached plug. 39 Consider the $x-z$ plane through the center of the plug (Fig. 3). Due to the beam shape, the initial concentration of excited unbleached fluorophores, $c_{0}$, within the plug immediately 41 after the photobleaching process, i.e. at $t=0$, is given by the Gaussian distribution presented in equation (1)

$$
c_{0}(x)=1-A e^{-\frac{\left(x-x_{0}\right)^{2}}{2 \sigma_{x}^{2}}}
$$

where $A$ is the fraction of bleached fluorophores, $x_{0}$ is the initial central position of the plug and $\sigma_{x}$ is the radius of the bleaching beam. Assuming steady state, homogeneous 
diffusion and incompressible flow, equation (2) describes the response of the initial

2 distribution of unbleached fluorsophores using a simplified convection-diffusion equation

$$
\frac{\partial c}{\partial t}=D \nabla^{2} c-\vec{u} \cdot \nabla c
$$

3 where $c$ is the spatiotemporal concentration of unbleached fluorophores, $t$ is time, $\nabla$ is the 4 gradient function, and $\vec{u}$ is the velocity field. The one-dimensional Reynolds equation can 5 be used to describe the flow in a thin film under isothermal conditions. A solution, using 6 no-slip boundary conditions, as presented in equation (3), shows that the through7 thickness velocity profile, $u(z)$, is a combination of a Poiseuille pressure-driven term (left) 8 and a Couette shear driven term (right)

$$
u(z)=\frac{1}{2 \mu} \frac{\partial P}{\partial x}\left(z^{2}-z h\right)+U \frac{Z}{h}
$$

9 where $\mu$ is the viscosity, $\partial P / \partial x$ is the pressure gradient, $h$ is the film thickness and $U$ is 10 the relative sliding speed. In the region of the contact where the film thickness is almost 11 constant, the flow is modelled as shear driven plane Couette with a pressure gradient.

12 The Poiseuille term is relatively small due to the high viscosity [23], so that

$$
u(z) \approx U z / h
$$

13 If a slip boundary condition is used instead at the stationary surface, the resulting velocity 14 profile is

$$
u(z)=U(z+b) /(h+b)
$$

where $b$ is the slip length.

16 The Peclet number $P e=L U / D$, where $L$ is the characteristic length and $D$ is the diffusion 17 coefficient of the fluorescent dye in $\mathrm{PB}$, describes the relative effects of convection and 18 diffusion. For the $z$ direction, where diffusion effects are largest in a thin film, the Peclet 19 number is $P e_{z}=h U / D$. The evolution of the intensity distribution is heavily dependent on 20 this number. If convection is favoured, the intensity distribution will vary in $z$. If diffusion 21 dominates, the distribution will be invariant in z. For experiments in this work the Peclet 22 number was above 1000. Molho et al. [27] concluded that if $P e>>100$, diffusion and 23 dispersion effects are negligible. As $P e_{x}=P e_{y}>>P e_{z}$, diffusion can be neglected 24 altogether. This enables the velocity profile reconstruction scheme to be simplified. The 25 fluid can be modelled as infinitesimal layers of concentration $c_{0}$ at $t=0$. Each layer 26 moves independently with velocity $u(z)$ and there is no mixing between layers. This is 27 shown schematically in Fig. 4a. 

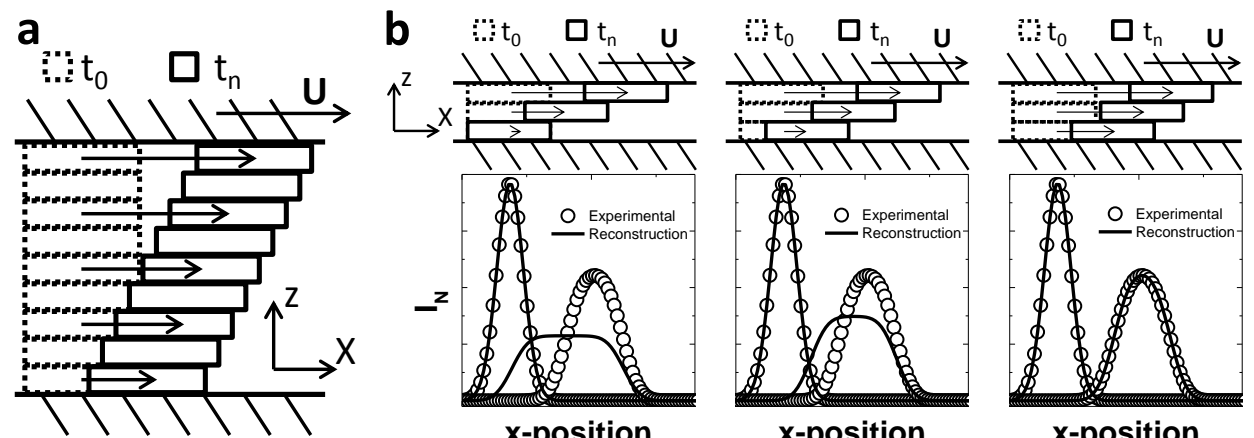

x-position

x-position

Fig. 4 Schematic of the framework used for the velocity profile reconstruction. (a) The fluid in the $x-z$ plane, modelled as layers moving independently of each other. (b) Top: Three successive guesses for the velocity profile at times $t_{0}$ and $t_{n}$. Bottom: Corresponding comparison between typical experimental (circles) and numerical (line) intensity distributions $I_{N}$.

Under the assumption that the photobleaching occurs relatively quickly, $c_{0}$ will be equivalent for all layers. The appropriateness of this assumption can be confirmed by studying the symmetry of the intensity distribution of the photobleached plug right after the photobleaching process. If the bleach time is relatively long compared to the velocity of the lubricant flow, the distribution will be distorted and appears non-Gaussian. Simulations performed using the conditions in the experimental work confirm that the effect of the bleach times used in this work on the reconstructed velocity profile was negligible.

Taking $\vec{u}=(u, 0,0)^{T}$ and using the assumption of negligible diffusion, equation (2) reduces to

$$
\partial c / \partial t=\vec{u} \cdot \nabla c=u \partial c / \partial x
$$

The solution to this hyperbolic partial differential equation is

$$
c(x, t)=c_{0}(x-u t)
$$

Using equation (1) and (7) the response of each layer can be described as

$$
c(x, z, t)=1-A e^{-\frac{\left(x-\left(u t+x_{0}\right)\right)^{2}}{2 \sigma^{2}}}
$$

Given a velocity profile $u(z)$ the theoretical spatiotemporal intensity distribution $I^{*}$, comparable to the experimental results, is the integral of these layers

$$
I^{*}(x, t)=\int_{0}^{h} c(x, z, t) d z
$$

This is in contrast with earlier experiments for similar submicron geometries [26,30], where significant diffusion reduces the technique resolution to two dimensions due to the equilibration of intensity in the z-direction. Variations of the intensity distribution in $z$ have previously been studied $[29,31]$ for larger dimensions, with the expected non-Gaussian response. The benefit of negligible diffusion in this work is that effects such as slip and inhomogeneous shear can be directly visualised, despite the narrow geometry of a contact. 
The experimental technique described in section 2 captures the evolution of the intensity distribution of a photobleached plug as the lubricant is being sheared. The analysis presented in section 3.1 correlates such evolution with the velocity profile of the lubricant through the determination of through-thickness concentration of unbleached probes. Most of the confined lubricant film, especially in regions away from the minimum film thickness region (horseshoe region), has constant film thickness, as confirmed in this work using interferometry. If the observation volume is small relative to the dimensions of the contact, the velocity profile can be assumed to be locally invariant in the $\mathrm{x}$-direction. Given that the total fluorophore concentration is constant and fluid motion is dominated by convection, the intensity distribution is directly coupled to the velocity profile such that $I(x, y, t)=f\left(I_{0}, u(z)\right)$. Unfortunately the coupling is non-trivial. It has previously been solved numerically by assuming an $\mathrm{n}^{\text {th }}$ order velocity profile [39]. It has also been solved by constraining the velocity profile [31]. A similar approach to [31] is used in this work. $u(z)$ is solved numerically by a least squares minimization of the difference between the theoretical intensity distribution $I^{*}$ as shown in equation (9) and the experimental distribution $I$. For the discretized intensity distribution, as is the case for the experimental data, the Cartesian axes $\mathrm{x}, \mathrm{y}$ and $\mathrm{z}$ are referred to as $\mathrm{i}, \mathrm{j}$ and $\mathrm{k}$ respectively and time $t$ is referred to as frame number $n$. To simplify the problem and to reduce noise, the intensity is averaged in the $y$-direction over $2 w$ pixels such that $I(i, n)=\sum_{j=-w}^{w} I(i, j, n) /(2 w)$. The minimization effectively varies the velocity profile until $I^{*}$ matches $I$. This is shown schematically in figure Fig. 3b, where the theoretical intensity distribution for slip Couette flow is to be reconstructed. Three successive iterations of the minimization are shown culminating in a match of the intensities and a determined velocity profile.

The discrete minimization takes the form

$$
\sum_{i=1}^{128} \sum_{n=1}^{N}\left\|I^{*}-I\right\|^{2}<\delta_{I}
$$

where $N$ is the number of images in the sequence and $\delta_{I}$ is the convergence criterion for the intensity minimization. It is assumed that the velocity of the fluid at the glass surface is not negative and, at the steel surface, the fluid does not flow faster than the surface speed $U$. This sets the boundary conditions $u(1) \geq 0$ and $u(h) \leq U$. While this scheme effectively finds the velocity profile that results in the best agreement between $I^{*}$ and $I$, the solution may not be unique. A second constraint is applied, which states that the velocity profile must be continuous and minimises the difference between velocities of adjacent lubricant layers. A second order finite difference scheme is chosen to impose this constraint

$$
\sum_{k=2}^{K-1}\|u(k+1)-2 * u(k)+u(k-1)\|<\delta_{u}
$$

where $K$ is the number of discrete layers used in the simulation and $\delta_{u}$ is the convergence criterion for the continuity minimization. Given $\delta_{I}$ and $\delta_{u}$, the $u(z)$ obtained

37 is unique. Thus the solution is affected by the minimization ratio $\delta_{I} / \delta_{u}$. After imposing a convergence criterion of $10^{-8}$ the complete minimization scheme is given by 


$$
\sum_{i=1}^{128} \sum_{n=1}^{N}\left\|I^{*}-I\right\|^{2}+\frac{\delta_{I}}{\delta_{U}} \sum_{k=2}^{K-1}\|u(k+1)-2 * u(k)+u(k-1)\|<10^{-8}
$$

1 This problem is solved using a non-linear least squares solver 'Isqnonlin' in Matlab. The 2 minimisation ratio was chosen based on two parameters which quantify the accuracy of 3 the reconstructed velocity profiles. The coefficient of determination $R^{2}$ can be used to 4 describe the goodness of fit that the simulated intensity $I^{*}$, produced by the reconstructed 5 velocity profile, has with the experimental intensity $I$. The standard error $\sigma_{s}$ of the 6 determined velocity of each layer is calculated based on the distribution of velocities 7 obtained in each iteration during the reconstruction of a velocity profile and can be 8 obtained by using the residuals and jacobian of the minimization problem [40]. The best 9 trade-off between a good intensity fit and a continuous profile is made when the ratio of

10 the two parameters $R^{2} / \sigma_{s}$ is maximised. This approach is consistent with other numerical 11 solutions investigating similar problems in that they either specify a velocity profile [39], or 12 a user defined minimization parameter [31]. The method of velocity profile reconstruction 13 employed in this study means that the number of layers $m$ does not affect the resolution 14 of the technique. Increasing $m$ only determines the number of points describing the 15 velocity profile, hence $m$ was kept constant for all cases in this study. A Matlab script

174 Validation

\subsection{Setup}

To assess the suitability of the use of photobleached imaging and the implementation of the velocity profile reconstruction algorithm for velocity profile determination, a flow with a known velocity profile was studied. Plane Couette flow was applied to a thin film of PB H300 between two glass microscope slides. Experiments were carried out for a film with 1 $\mu \mathrm{m}$ thickness. This film thickness is below the depth of focus of the optical system when a magnification of $20 \mathrm{x}$ is used, ensuring a through-thickness photobleached plug can be formed by photobleaching. A constant velocity was applied to the top slide while the bottom slide remained stationary, creating the Couette flow in the PB film.

\section{$27 \quad 4.2$ Results}

The experimental and reconstructed spatiotemporal intensity distribution of plane Couette flow in a $1 \mu \mathrm{m}$ PB film is presented in Fig. 5a. The velocity of the top slide was $26.3 \mu \mathrm{m} / \mathrm{s}$ while the bottom slide remained stationary. The bleach time was $25 \mathrm{~ms}$, the exposure time was $180 \mathrm{~ms}$. The resulting photobleached plug had an initial diameter of $20 \mu \mathrm{m}$. 10x synchronous averaging was applied to improve the signal to noise ratio of the result. Single acquisitions were also carried out, yielding similar results. 

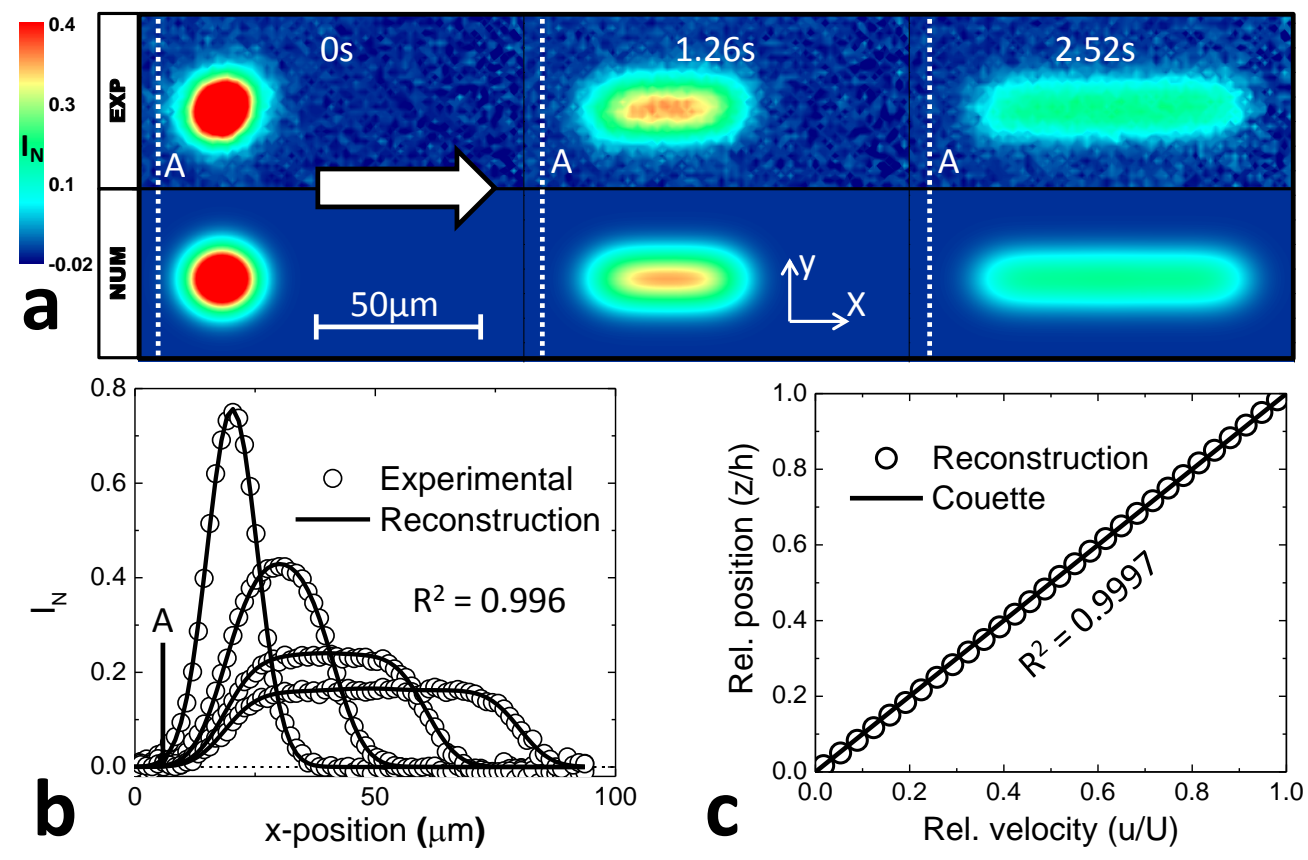

Fig. 5 Plane Couette validation results. (a) Experimental (top) and reconstructed numerical (bottom) spatiotemporal intensity distribution. The white arrow indicates the shearing direction of the fluid. (b) Comparison between averaged experimental (circles) and numerical (line) intensity distributions. (c) Reconstructed (circles) and Couette (line) relative velocity profiles.

Compare the simulated image sequence (the 'NUM' row in Fig. 5a) with the experimental result (the 'EXP' row in Fig. 5a), the reconstructed distribution agrees well with the experimental results in the evolutions of both intensity and shape of the photobleached plug. The integral of the intensity distribution varies less than $2 \%$ over the sequence. To better quantify the goodness of fit between $I^{*}$ (numerical) and $I$ (experimental), the data was analyzed further by averaging the intensity in y over 5 pixels. The resulting intensity distribution is shown in Fig. 5b. The initial intensity distribution of the photobleached plug is well approximated by a Gaussian distribution $\left(R^{2}>0.99\right)$, showing that the bleaching process can be assumed to be instantaneous. The average coefficient of determination of all fits is $R^{2}=0.996$, supporting a good agreement between the numerical reconstruction and the experimental data. Both Fig. $5 \mathbf{a}$ and Fig. $\mathbf{5 b}$ show no change in the position at the back of the photobleached plug (point $A$ in Fig. 5b) with time, suggesting no measurable slip can be observed. The intensity distribution flattens out at longer times, which is expected for Couette flow.

The reconstructed velocity profile (shown as circles) in Fig. $\mathbf{5 c}$ matches very well with the Couette flow (black line) applied to the PB film. In addition, correct surface velocities for both top and bottom plates are found. Thus it justifies the capability of the velocity profile reconstruction algorithm in obtaining accurate velocity profiles. These results also confirm that the experimental framework and the assumption of negligible diffusion (stated in section 3.1) are applicable to these experimental conditions.

Conclusively, using appropriate assumptions, it is possible to use the two-dimensional spatiotemporal intensity distribution to probe velocity variations through the thickness of a thin film. Having validated the use of the proposed technique for a simple flow, it was applied to the case of EHL. 
 \\ 5 EHL flow profiling}

\subsection{Setup}

A pure sliding contact was created by loading a $3 / 4$ " rotating steel ball (PCS instruments) onto a microscope glass slide, with a polydimethylsiloxane bath containing the PB (see Fig. 2). The roughness of the ball and glass slide was measured using a Veeco optical profilometer in phase-shifting interferometry mode. The arithmetic mean value $R a$, was found to be less than $5 \mathrm{~nm}$ for the steel ball and less than $0.5 \mathrm{~nm}$ for the glass slide. The location of the ball was fixed using a ball carriage assembly (PCS instruments) that constricted the motion in the z-direction. A load of $5 \mathrm{~N}$ was applied using a dead weight. This produced a theoretical Hertzian contact of radius $87 \mu \mathrm{m}$ which was confirmed experimentally by fluorescence imaging and interferometry. Fig. 6 shows the EHL contact visualised using fluorescence imaging at 10x magnification. The maximum theoretical Hertzian pressure at this load was $315 \mathrm{MPa}$. The surface velocity was calibrated by tracking features on the steel ball surface and was found to be accurate within $2 \%$. The central $x$ - and $y$-position of the contact was maintained within $5 \mu \mathrm{m}$ over the revolution of the steel ball. Compare the experimental time frame $(\sim 100 \mathrm{~ms})$ to the characteristic time of contact drift ( 166 s), such drift will be ignored in this study. An EHL ultra thin film measurement system (PCS instruments) was used to measure the film thickness using interferometry. Most of the film within the contact had a constant thickness, allowing the flow in the hatched region in Fig. 6 to be analysed as plane Couette flow. The steel ball was driven by a stepper motor. The sliding velocity was $360 \mu \mathrm{m} / \mathrm{s}$, giving a film thickness of $170 \mathrm{~nm}$. The sliding speed and load were chosen such that the contact operated within the piezoviscous-elastic region. The mapping coordinates, indicating the positions at which the fluid was probed, are indicated by white crosses.

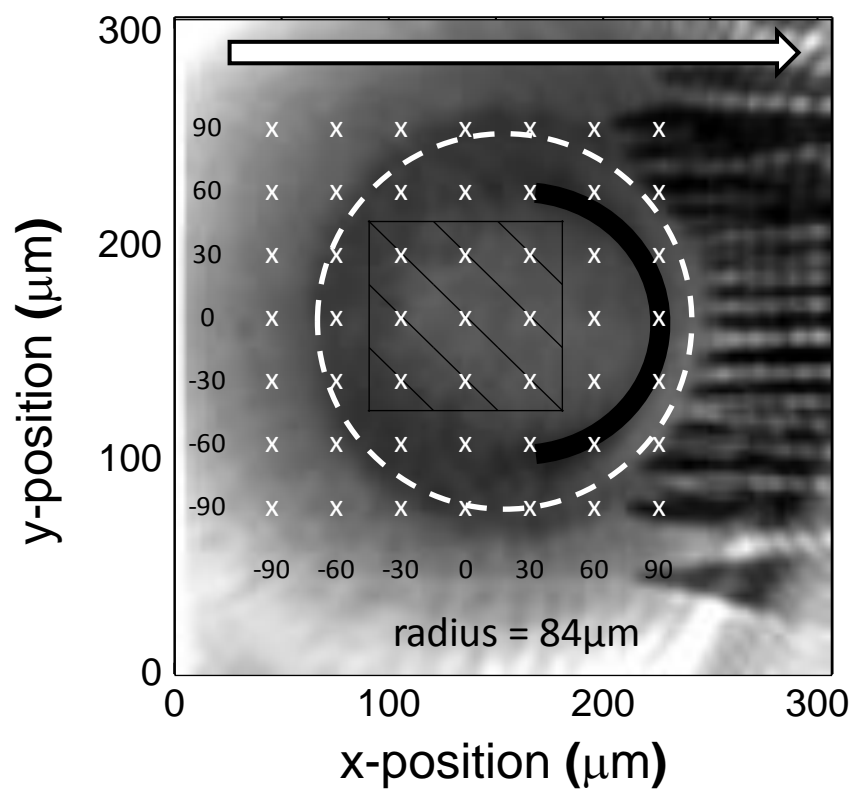

Fig. 6 Fluorescence image of the EHL contact. Brighter intensity generally corresponds to a thicker film. The flow direction is indicated by the white arrow. The calculated Hertzian contact area is marked by the dotted white line. A grid used for mapping the slip length and flow direction in the contact is shown overlaid with distance referenced to position $[0,0]$, given in microns. The horseshoe is indicated by a black arc. 
The temperature of the fluid needs to be considered as a change in just a few degrees significantly alters the viscosity, and therefore the velocity profile. Heat can be generated due to (1) friction; and (2) photobleaching. In contacts, temperature rises due to friction of up to $50^{\circ} \mathrm{C}$ have been measured experimentally [41]. The expected temperature rise can be determined using analysis described in [1]. Using a conservative friction coefficient of 0.1 [42] and the thermal properties for PB [43], the temperature rise due to friction in the applied experimental condition was estimated to be less than $0.01 \mathrm{~K}$; the low value being primarily due to the very low sliding speeds employed. The temperature rise for the same system has been assumed to be negligible by others [44]. It should also be noted that, if the temperature rise was significant, there would be a distinct difference in the velocity profile near the two surfaces due to the difference in heat transfer. This was not seen experimentally. The effect of the temperature rise due to photobleaching can be neglected, as shown in section 2.4. Thus isothermal conditions were assumed in this study.

\subsection{Results}

Photobleached imaging with 100x synchronous averaging was applied to an EHL point contact. The bleach time was $8.5 \mathrm{~ms}$, the exposure time was $4 \mathrm{~ms}$. A 50x objective was used for imaging, resulting in a photobleached plug with an initial diameter of $6 \mu \mathrm{m}$. The first two images acquired for each set of experiments are not analyzed due to the finite opening time of the shutter protecting the camera from the bleach beam. Therefore, results are shown after this shutter has completely opened. In all experiments, the integral of the experimental intensity distribution varied no more than $2 \%$ while the whole plug was contained within the observation area. A typical experimental result obtained at a position close to the center of the EHL contact (position [0, 0] in Fig. 6), is presented in Fig. 7. The numerical intensity distribution computed based on the velocity profile reconstruction algorithm is also included for comparison. The averaged intensity distribution over 5 pixels in the y-direction is shown in Fig. 7b.

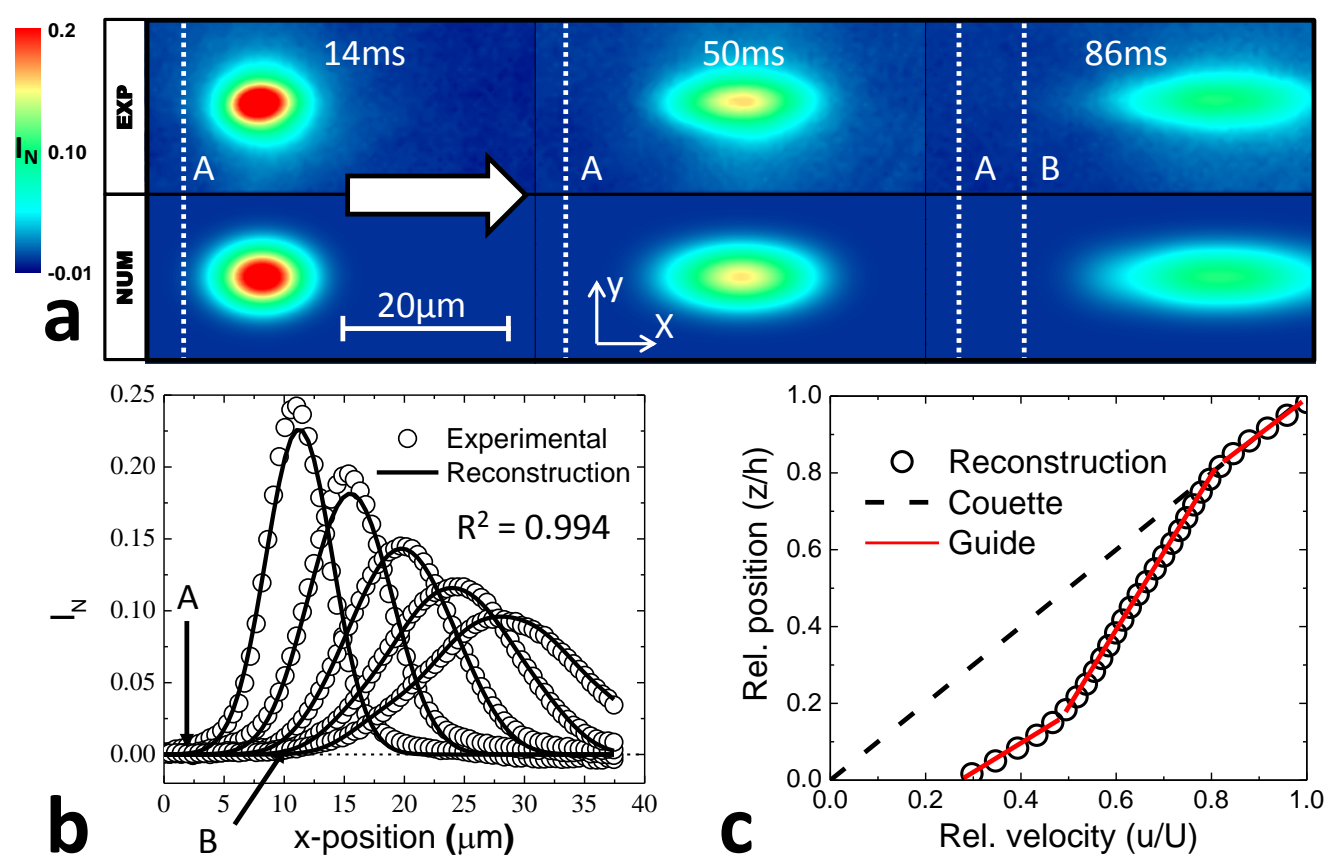

Fig. 7 Photobleached imaging velocimetry results for an EHL contact. (a) Experimental (EXP) and reconstructed numerical (NUM) spatiotemporal intensity distribution. The white arrow indicates 
the shearing direction of the fluid. (b) Comparison between averaged experimental (circles) and

2 numerical (line) intensity distributions. (c) Reconstructed (circles) and Couette (dashed line)

3 relative velocity profiles. The solid lines are for guidance and indicate that the lubricant was

4 separated into three distinct layers.

5 The initial intensity distribution, as shown in Fig. $\mathbf{7 b}$, was symmetric, which confirms that 6 the assumption of instantaneous bleaching is appropriate. A comparison between results 7 obtained in an EHL contact (Fig. 7) and under the no-slip Couette condition (Fig. 5), as 8 applied in the validation experiment, reveals several anomalies. Significant motion of the 9 back of the spot, from point A to point B as indicated in Fig. 7b, was observed, suggesting 10 that slip has taken place at the bottom surface. This is confirmed by the reconstructed 11 profile in Fig. 7c with PB having a relative velocity of about 0.3 at the stationary glass 12 surface. Secondly, at longer times, the photobleached plug only elongated by a small 13 amount and the average intensity distribution did not show a plateau as in the case of the 14 Couette flow (Fig. 5b). Therefore, the use of a slip Couette fit to describe the velocity of the system studied under EHL condition would be inappropriate.

The reconstructed velocity profile based on the proposed velocity profile reconstruction scheme is presented in Fig. 7c. This is the first published result of a through-thickness velocity profile measurement in an EHL contact. It shows that the velocity profile at this particular location is non-linear. The resulting numerical intensity distribution matched the experimental results well, with an average goodness of fit $R^{2}=0.994$ (shown in Fig. 7b). Therefore, the reconstructed, nonlinear velocity profile provides a satisfactory account of the experimental intensity response. This non-linear velocity profile contradicts the profile commonly assumed in EHL theory [1,2], and those found using isothermal simulations [45]. It suggests that the fluid experiences different shear rates depending on the distance from the rubbing surfaces. While the physical cause for the shape of the profile is not yet known, such that the mathematical form that should be adopted for the profile is unclear; one can still clearly identify three regions through the thickness of the film, as indicated by the overlaid solid lines. Layers with localized shear rates appear to have developed within the fluid, with the surface regions experiencing higher shear rates than the lubricant in the central region.

\section{Mapping}

With a method for determining velocity profiles established, the technique is applied to various positions in an EHL contact to investigate flow heterogeneities. The onedimensional theoretical normal pressure experienced by the lubricant in an EHL contact can be determined using Hertzian contact mechanics. Since a point contact is used, there is also a significant pressure gradient in the y-direction resulting in two-dimensional flow. The position-dependent pressure and the geometric effect may cause the flow profile of the lubricant to vary throughout the contact. It is possible that non-linear velocity profiles observed in this study may result from a pressure induced viscosity change in the liquid. If this is the case, there should be significant variations in both slip length and velocity gradient with pressure. This can be verified by mapping the velocity profiles in the contact. Measurements of the through-thickness velocity profile and the 2-D $x-y$ plane average velocity were performed at positions marked as ' $x$ ' in Fig. 6 within a $7 x 7$ grid. Six tests were carried out at each position with different specimen, and average values are reported. 


\section{$2 \quad 6.1$ Two-dimensional flow map in the $x-y$ plane}

3 The two-dimensional flow of a lubricant in a contact is complicated, due to the unknown 4 pressure distribution and the rheology of the liquid. There are simultaneous effects of 5 geometry, cavitation and pressure gradients. The spatiotemporal intensity maps can be used to characterise the z-averaged two-dimensional flow of the liquid. The $y$-component

7 of velocity, at time $t$, was determined by finding the position of the center of mass of 8 intensity $y_{C M}$ using

$$
\int_{y=0}^{W}\left(y \int_{x=0}^{L} I(x, y, t) d x\right) d y=\left(y_{C M}+y_{0}\right) \int_{y=0}^{W} \int_{x=0}^{L} I(x, y, t) d x d y
$$

9 where $W$ is the width (in the y-direction) of the observation area, $L$ is the length (in the $\mathrm{x}$ 10 direction) of the observation area and $y_{0}$ is the central position of the initial bleach spot in 11 y. $y_{0}$ is found by fitting a two-dimensional Gaussian intensity distribution to the initial spot 12 using

$$
I_{0}(x, y)=1-A e^{-\left(\frac{\left(x-x_{0}\right)^{2}}{2 \sigma_{x}^{2}}+\frac{\left(y-y_{0}\right)^{2}}{2 \sigma_{y}{ }^{2}}\right)}
$$

where $\sigma_{y}$ is the radius in y of the bleaching beam. Equations (13) and (14) are discretized to allow the use of the pixelated experimental data. The average velocity of the lubricant is then given by $y_{0} / t$. A similar expression was used to determine the $x$ component of velocity. Fig. 8 shows a vector plot of the two-dimensional velocity profile within the EHL contact and in the inlet region. For clarity, the $y$-component of the vectors shown has been scaled up tenfold.

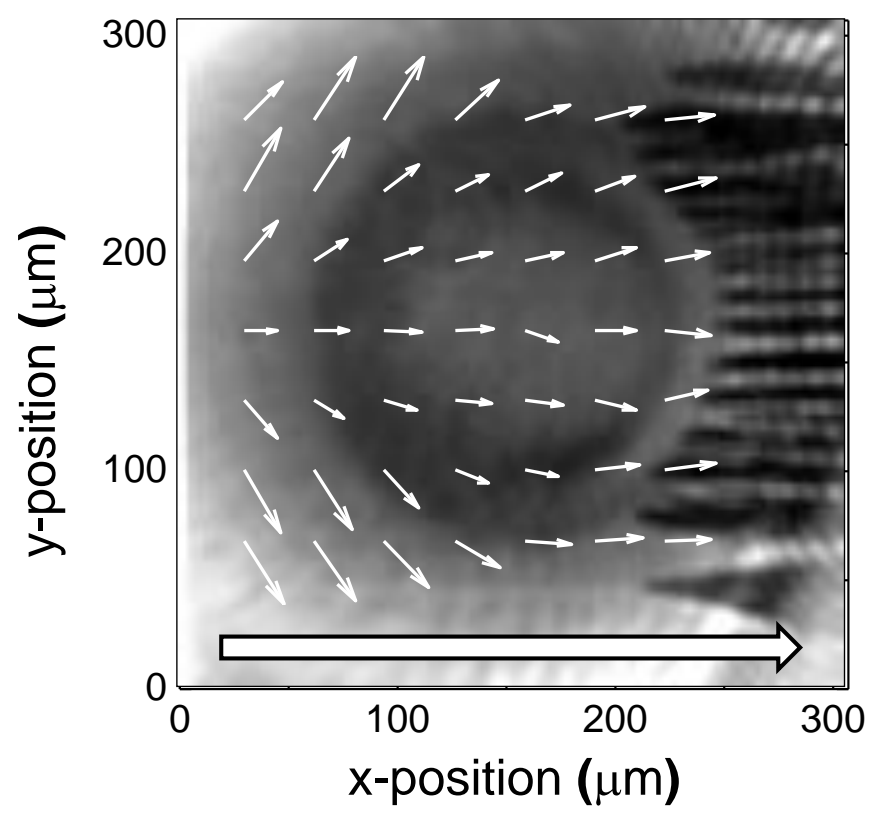

Fig. 8 Fluorescence image of the contact. Vector plot of velocity field overlaid with white arrows. 
The $x$-component was much larger than the $y$-component in all positions. Within the contact, the $y$-component of velocity was less than $5 \%$ of the $x$-component. Due to the high viscosity of pressurised $\mathrm{PB}, \frac{h^{2}}{\mu} \frac{\partial P}{d x}$ is relatively small. Thus, based on equation (3), the Poiseuille component of flow is effectively negligible. In the central region of the contact, the flow in the y-direction was minimal. Near the inlet however, geometry effects play a much larger role, causing the liquid to flow around the contact. At the outlet, the results are distorted due to cavitation.

\subsection{Slip measurements}

The existence of 2-D flow, while small, does have an effect on the quality of the reconstructed velocity profile. Furthermore, near the edges of the contact, the film thickness is not constant. Only reconstructed profiles with a goodness of fit, $R^{2}$, higher than 0.99 are used for further analysis. In the central region of the contact (hatched area in Fig. 9), flow in the $y$-direction was minimal, and the film thickness was constant to give reconstructed velocity profiles with $R^{2}>0.99$. The glass-PB interfacial velocity was determined using a linear fit. The fit was made using the first three points of the velocity profile from $z=0$ (as indicated by the bottom solid line in Fig. 7c), and the slip velocity was determined by extrapolation to $z=0$. The slip length was then found using equation Error! Reference source not found.. The slip length as a function of position is shown in Fig. 9a and Fig. 9b.
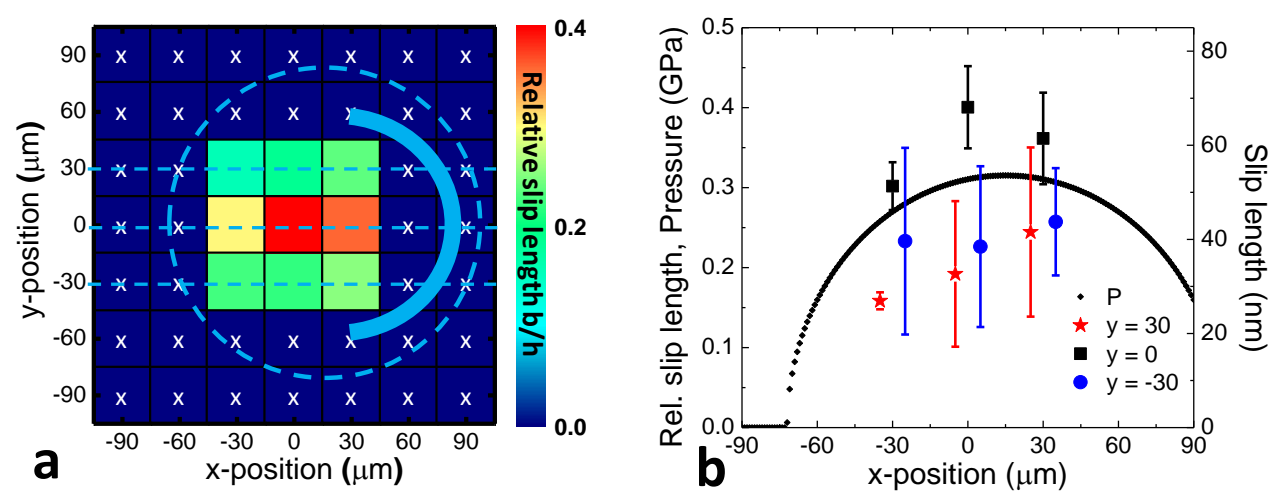

Fig. 9 Variation of slip length with position in the contact. (a) Color map of relative slip length against the position in the contact. The Hertzian contact area (dotted circle) and the horseshoe (thick arc) are overlaid. Crosses indicate positions with $R^{2}<0.99$. The center lines of the selected rows are indicated by straight dotted lines. (b) Mean relative slip length along the three center lines in (a) with respect to position in $\mathrm{x}$. All data points are at $x=-30,0$ or $30 \mu \mathrm{m}$. The data for $y=30$ and $-30 \mu \mathrm{m}$ has been shifted in $\mathrm{x}$ for clarity. The pressure for the central line $(y=0)$ is also plotted against $x$-position in the contact. The error bars indicate the standard deviation of the measured slip length for the results with $R^{2} \geq 0.99$.

There is a significant variation of the relative slip length (measured slip length divided by the film thickness) with respect to the probing position in the contact, as shown in figure Fig. 9. Slip lengths of $30-70 \mathrm{~nm}$ have been measured at the PB-glass interface. Since the theoretical Hertzian pressure also varies with position in the contact, the normal pressure experienced by the fluid and the slip length measured may be correlated. As discussed in section 5.2, the typical flow profile as shown in Fig. 7 can be divided into three regions: two regions next to the rubbing surfaces with high shear rate, and the 
central region with a lower shear rate. Apart from the changing slip length, a variation in shear rate experienced by the central region of the flow profiles against $x$-position is also observed. Since both a change in viscosity due to normal pressure and a change in interfacial slip can affect the relative shear rate, it makes the two effects difficult to decouple. More work is in progress to establish their relationship.

7 Discussion

The methodology presented in this work uses photobleached imaging and a minimization scheme to reconstruct the through-thickness velocity profile of a liquid film under $\mathrm{EHL}$ conditions. Slip lengths for ambient pressure conditions have been measured previously using photobleaching [24-26]. However the methodology developed in this study is the first that allows capturing velocity variations through submicron film thicknesses. It offers several distinct benefits. The depth of focus of the bleaching beam, which determines the thickest fluid film the bleaching beam is able to form a through-thickness photobleached plug for velocity profile reconstruction, is about $1 \mu \mathrm{m}$. This makes the technique particularly suited for the study of submicron EHL films. The method can also be extended to investigate the flow of confined liquid films with a similar range of thicknesses. The through-thickness velocity profiles obtained contain information about the flow properties of the sheared lubricant. The relative shear rate at every z-position can be obtained using the slope of the velocity profile. If the shear stress is known, the through thickness viscosity variation of the liquid can be determined. Hence the technique would enable in situ rheometry [46]. The non-monotonic stress-strain relationship as indicated by the existence of multiple shear rates at a given shear stress would not have been captured by conventional friction measurements where only average observations can be made.

The approach developed in this study is not able to resolve sharp velocity gradients near the wall, although molecular dynamics results suggest that such variations are not prevalent [13,47-49]. This is because the experimental result is an average of the intensity within the film, and the relative intensity of one or two molecular layers at the proximity to the wall would be insignificant. In addition, the velocity profile reconstruction requires the fluid to be composed of layers of finite thickness which should not be thinner than the size of an individual fluid molecule. Thus the interfacial velocity will be measured at minimum one fluid layer away from the surface, however thin this layer is. Although it is possible to measure the velocity of the fluid very close to the interface, the true interfacial slip velocity cannot be resolved unless the correct mathematical form of the flow profile is known. The nature and the amount of the slip, whether it is apparent or real, are therefore not precisely determined in this work. While this is a drawback, it is also a distinction other techniques are unable to make. A continuity criterion imposed on the velocity profile reconstruction scheme, as stated in equation (12), limits the relative shear rates that can be measured with the proposed technique. The proposed approach will still capture discontinuities in velocity profiles when the shear rate approaches zero, although such profiles will be smoothed. Thus the technique is capable of detecting shear localization at the interface (slip) and at the center of the film. Implementation of other criteria to alleviate this issue is possible and is in progress. Nevertheless the profile obtained is valuable for better understanding the rheological behaviour of the fluids which is essential in developing the necessary model for obtaining the interfacial velocity and viscosity, and hence the local friction.

47 The technique works best when the diffusion of the fluorescent probe through the 48 thickness of the film is small, i.e when the Peclet number is large. Low Peclet number 49 conditions can be accommodated to an extent by incorporating the effect of the through50 thickness diffusion of fluorescent probes in the velocity profile reconstruction scheme. 
This will be implemented in future work. If the Peclet number is small, the technique can also be used to measure diffusion coefficients using an alternative analysis [30].

The interfacial slip length determination presented in this work, unlike most slip length measurements carried out in EHL contacts $[16,17]$ which assume a linear velocity profile, does not require knowledge a priori of the through-thickness flow profile. Based on the results found here, the assumption of a linear velocity profile in EHL contacts can be inappropriate and hence the estimations of slip velocity, and the slip length erroneous. The relaxation on the flow profile assumption opens up the possibility of applying other possible profiles in an EHL contact and is crucial to the understanding of rheology in such conditions.

The proposed methodology allows point-wise measurements, such that velocity profiles can be measured at different positions in the contact. This effectively creates a threedimensional velocity profile map and allows local rheological properties of the lubricant to be investigated. This differs from commonly-used friction-based and interferometry-based techniques which infer the nature of the lubricant flow properties from ensemble measurements. The mean slip length across the contact has been previously measured using interferometry [17] by observing the motion of the dimple created in the EHL film. Yet, the successful mapping of flow profiles in the contact, and the location dependent velocity profile and slip length presented in this study demonstrate that heterogeneous behaviour can exist in a contact. Hence the constant slip length usually assumed for modelling friction reduction by interfacial slip in a contact $[16,17]$ can be an inappropriate assumption and requires verification. The relationship between local slip and friction, being an ensemble average measurement, is more complicated than the conventional treatment might suggest. This, along with the non-linear profile, could possibly explain the anomalous Stribeck curves found [42] for the same system. More experiments are required to understand the relationship between slip length, normal pressure and shear rate. This is not a trivial undertaking as the viscosity varies non-linearly with pressure and shear rate [3], which in turn influences the shear stress experienced by the sheared fluid. Furthermore, the interfacial shear stress affects the slip length for polymers in a nonlinear manner [24].

The finding that the velocity profile is non-linear as presented in Fig. 7c, in other words, the existence of inhomogeneous shear for an isothermal case, is of significance. Similar profiles have been observed for flows with significant temperature gradients [50,51]. Inhomogeneous shear has also been observed for heterogeneous fluids [9-11]. The pressure sensitive rheology of $\mathrm{PB}$, as described in section 2.4, may contribute to its peculiar velocity profiles. It is conjectured that the pressure causes the lubricant to solidify. Energy is then somehow dissipated and causes temperature rises sufficient to melt the layers near the two boundaries and thus the lubricant flows like a plug in the center. In the current work, the existence of a definite shear rate in the central region of the lubricant, as shown by the velocity profile in Fig. 7c, indicates that the photobleached plug did not completely solidify. One explanation for the behaviour is that rather than complete solidification, the glass transition temperature of the fluid is increased under the experimental conditions and hence the viscosity increases substantially. Indeed in some instances, liquid can undergo a second order phase transition [52]. Surface heating can reduce the viscosity of the fluid near the surfaces, yet the mechanism of this effect is unknown. The heat from photobleaching is low $(3 \mathrm{~K})$ and decays too quickly (in $1 \mathrm{~ms}$ ) to have an effect. While friction induced heating is a candidate, the insignificant theoretical temperature rise of $0.01 \mathrm{~K}$ suggests the contrary. Hence the formation of a temperature gradient is unlikely. In addition, if the heating were friction induced, the heat transfer at the two surfaces would be significantly different and so would the shear rates, which is not the case. The source of possible surface heating and hence the origin of displayed 
variations of shear rate and/or viscosity across the thickness of the film remains unclear. There is currently no theoretical description of the velocity profile encountered in this work, making a comparison to other work cumbersome. Similar profiles to those presented in this work have been observed experimentally in systems with pressures and shear rates comparable to EHL $[13,53]$ and in molecular dynamics simulations of amorphous systems [54]. They have also been suggested for the EHL condition [7]. Phase-changes can cause inhomogeneous shear and would be expected for a liquid of the viscosity used in this work [6]. Under the effect of shear, solidification should cause significant changes in the stress and viscosity field and thus the velocity profile might deviate from the linear shape predicted by the Reynolds equation. To further clarify the effect of solidification on velocity profiles, a study investigating the influences of imposed shear rate and normal pressure on the formation of inhomogeneous layers should be carried out. This is a topic for future work. Experiments with low viscosity lubricants to investigate the existence of similar phenomena at high shear rates will be carried out.

In EHL theory [1,2], the friction at any point in the contact is given by the throughthickness integral of the shear stress. It is normally assumed that the shear rate is constant (i.e. Couette flow). This allows the determination of the shear stress through many of the available relationships that relate shear stress $\tau$ to shear strain $\dot{\gamma}$ using viscosity $\mu: \tau=f(\mu, \dot{\gamma})$. If the shear stress experienced by the lubricant is constant throughout the film, the variation of the local shear rate through the thickness of the film presented in this work implies that local viscosity heterogeneity exists. This means that current EHL theory, which uses an average shear rate, is inadequate in accurately predicting the viscosity in situations where inhomogeneous shear is prevalent. Thus, the film thickness and friction predictions are invalidated as well. This also applies to any analysis requiring knowledge of the flow profile, such as infrared temperature measurements [12] used to determine surface stresses.

Data on through-thickness velocity profiles in thin films are scarce due to the experimental challenge imposed by the thickness of EHL films. Through-thickness flow profiles have been examined using similar molecular tagging velocimetry techniques under Poiseuille flow conditions [31,39]. These results were however obtained with films thicker than those encountered in EHL conditions, and thus may have little direct relevance in EHL. In addition, the rate of diffusion in their systems could potentially be prohibitively fast $[26,30]$ to be applied to the determination of flow profiles in a thin film. Therefore the results presented in this paper, while performed with a model system, are unique and show that the methodology developed is capable of studying the flow of an EHL film. While the details of the profile will obviously be governed by properties of the fluid observed, the applicability of the approach is independent of the materials system used.

The spatial resolution of the technique is on the order of $30 \mu \mathrm{m}$ in the direction of flow (xdirection and about $2 \mu \mathrm{m}$ normal to the flow (y-direction). These values are defined as the minimum observation area required for the velocity profile reconstruction scheme to make good fits $\left(R^{2}>0.99\right)$. For the slip velocity measurement, the resolution is approximately $20 \mu \mathrm{m} / \mathrm{s}$ (determined by simulations testing the ability of the reconstruction scheme to reproduce known velocity profiles) and thus about $10 \mathrm{~nm}$ for the slip length. The zresolution for velocity measurements is estimated to be at minimum two molecular layers $(\sim 5 \mathrm{~nm})$. It should be noted that the slip- and z-resolution cannot be directly measured due to the use of a reconstruction scheme to obtain the velocity profile. The real resolution could be found if known sub-micron velocity profiles were available for comparison. The temporal resolution of the technique currently allows capturing transient phenomena of about $15 \mathrm{~ms}$. With image accumulation and averaging, the signal to noise ratio of images increases and that allows better fitting of flow profiles. The temporal 
resolution can be significantly improved with the use of shorter photobleaching times (made possible with the use of mechanical chopper, and a more photosensitive dye [55]). This would allow flow profiles of lower viscosity lubricants to be examined. The resolutions of the technique compare well to other techniques. Both local slip length and velocity profile can be obtained by conducting point-wise measurements. The average slip length has been measured in an EHL contact by the dimple method [17]. Due to the large difference in lubricant film thickness it is difficult to compare the resolution of the slip length measurements between [17] and this work. Compared to slip measurements performed by fluorescence recovery after photobleaching [25] and by double-focus cross correlation [20] in other geometries, the slip length resolution is higher in this work, mainly due to the use of a thin lubricant film. In regards to stimulated emission depletion photobleaching anemometry [18] the spatial resolution in the x-direction and the temporal resolution for this work is relatively low at the moment but can be improved as discussed above. The resolution in $z$ is however enhanced, mainly because no z-positioning is required for this technique.

With the experimental and analytical protocol established, both the global and local effects of various parameters, such as base oil properties, operating conditions (pressure, entrainment speed and slide-roll ratio), and the use of additives on flow properties of the lubricant system can be studied. The knowledge obtained will be helpful for developing a new theoretical framework for EHL with inhomogeneous shear and will be useful for thin film lubrication applications as well as other nanoscopic flows.

\section{Conclusions}

A novel methodology, based on the use of photobleached imaging, is presented to allow the local through-thickness velocity profile and the slip length to be measured in an EHL contact. The first measurement of the through-thickness velocity profile in an EHL contact has been obtained in this work. The technique was first validated by successfully reconstructing the expected linear velocity profile without slip for a micron thick film of PB under Couette flow. It was then applied to an EHL contact where it was observed that PB slips on the glass surface and takes on a non-linear velocity profile. The drastic change of the velocity profile in the EHL contact cannot be caused by diffusion based on the large Peclet number for the EHL case. Assuming that the shear stress experienced by the lubricant remains constant throughout its thickness, the non-linear profiles obtained suggest a variation in through-thickness viscosity. The large normal pressure experienced by the lubricant causes an increase in the viscosity of the lubricant and possibly a phase transition and hence the lubricant flows almost like a plug in the central region of the film. Near the boundaries, the lubricant possesses lower viscosity which could be due to energy dissipation and/or surface heating. This causes the velocity profile to separate into three layers of varying shear rate, commonly referred to as inhomogeneous shear. Pressure induced inhomogeneous shear has been shown to be possible in theory and in molecular dynamics. It has also been seen in experimental measurements of thicker pressurised films and complex fluids.

Since the technique can obtain the local velocity profile in the contact with point-wise measurements, a velocity profile mapping was carried out. The results show that the local slip length in the contact varies with probing position. This can be related to the variation of pressure with location, and therefore the variation of the solid-liquid interfacial shear stress.

The effects of slip and inhomogeneous shear on film thickness, friction and the general 48 analysis in EHL applications are significant and therefore the study and development of 49 theories describing these phenomena are necessary. This can only be done with the 
assistance of experimental data which has until now been unavailable. While a viscous fluid is used as model lubricant, the technique can be extended to study lower viscosity lubricants by using a more photosensitive system and incorporating the effect of diffusion in the velocity reconstruction scheme. Ultimately, the technique will be useful for in situ rheology in EHL thin films and for velocimetry in submicron flows of viscous liquids.

Acknowledgements This study was supported by EPSRC Platform Grant (

7 EP/G026114/1). A.P. was sponsored by EPSRC DTA studentship. M.C. was supported

8 by EPSRC Grant no. EP/J008141/1. The authors would like to thank Johan Guegan for

9 interferometry measurements and David Heyes, Daniele Dini, Hugh Spikes and Luca di

10 Mare for fruitful discussions.

12 The authors declare that they have no conflict of interest

References

[1] Olver, A. V, Spikes, H. A.: Prediction of traction in elastohydrodynamic lubrication. Proc. Inst. Mech. Eng., Part J 212, 321-332 (1998)

[2] Gohar, R.: Elastohydrodynamics. World Scientific Publishing Company (2001)

[3] Bair, S.: The high-pressure, high-shear stress rheology of a polybutene. J. Non-Newtonian Fluid Mech. 97, 53-65 (2001)

[4] Bair, S., Jarzynski, J., Winer, W. O.: The temperature, pressure and time dependence of lubricant viscosity. Tribol. Int. 34, 461-468 (2001)

[5] Larsson, R., Andersson, O.: Lubricant thermal conductivity and heat capacity under high pressure. Proc. Inst. Mech. Eng., Part J 214, 337-342 (2000) under very high pressure by observing microsphere deformation and viscosity prediction. Lubr. Sci. 22, 417-429 (2010)

[9] Katgert, G., Möbius, M., Van Hecke, M.: Rate Dependence and Role of Disorder in Linearly Sheared Two-Dimensional Foams. Phys. Rev. Lett. 101, 3-6 (2008) 
[13] Heyes, D. M., Smith, E. R., Dini, D., Spikes, H. a, Zaki, T. A.: Pressure dependence of confined liquid behavior subjected to boundary-driven shear. J. Chem. Phys. 136, 134705 (2012)

[14] Mhetar, V., Archer, L. a.: Slip in Entangled Polymer Melts. 1. General Features. Macromolecules 31, 8607-8616 (1998)

[15] Vinogradova, O. I., Belyaev, A. V: Wetting, roughness and flow boundary conditions. J. Phys. Condens. Matter 23, 184104 (2011)

[16] Choo, J. H., Glovnea, R. P., Forrest, A. K., Spikes, H. A.: A Low Friction Bearing Based on Liquid Slip at the Wall. J. Tribol. 129, 611 (2007)

[17] Guo, F., Li, X. M., Wong, P. L.: A novel approach to measure slip-length of thin lubricant films under high pressures. Tribol. Int. 46, 22-29 (2011)

[18] Kuang, C., Wang, G.: A novel far-field nanoscopic velocimetry for nanofluidics. Lab Chip 10, 240-5 (2010)

[19] Li, H. F., Yoda, M.: Multilayer nano-particle image velocimetry (MnPIV) in microscale Poiseuille flows. Meas. Sci. Technol. 19, 075402 (2008)

[20] Vinogradova, O. I., Koynov, K., Best, A., Feuillebois, F.: Direct measurements of hydrophobic slippage using double-focus fluorescence cross-correlation. Phys. Rev. Lett. 102, 118302 (2009)

[21] Staben, M. E., Zinchenko, A. Z., Davis, R. H.: Motion of a particle between two parallel plane walls in low-Reynolds-number Poiseuille flow. Physics of Fluids 15, 1711 (2003)

[22] Piorek, B., Mechler, A., Lal, R., Freudenthal, P., Meinhart, C., Banerjee, S.: Nanoscale resolution microchannel flow velocimetry by atomic force microscopy. Appl. Phys. Lett. 89,153123 (2006)

[23] Reddyhoff, T., Choo, J. H., Spikes, H. a., Glovnea, R. P.: Lubricant Flow in an Elastohydrodynamic Contact Using Fluorescence. Tribol. Lett. 38, 207-215 (2010)

[24] Migler, K., Hervet, H., Leger, L.: Slip transition of a polymer melt under shear stress. Phys. Rev. Lett. 70, 287-290 (1993)

[25] Leger, L., Hervet, H., Massey, G., Durliat, E.: Wall slip in polymer melts. J. Phys. Condens. Matter 9, 7719 (1997)

[26] Schmatko, T., Hervet, H., Leger, L.: Friction and Slip at Simple Fluid-Solid Interfaces: The Roles of the Molecular Shape and the Solid-Liquid Interaction. Phys. Rev. Lett. 94, 244501 (2005)

[27] Molho, J. I., Herr, A. E., Mosier, B. P., Santiago, J. G., Kenny, T. W., Brennen, R. A., Gordon, G. B., Mohammadi, B.: Optimization of Turn Geometries for Microchip Electrophoresis. Anal. Chem. 73, 1350-1360 (2001)

[28] Herr, A. E., Molho, J. I., Santiago, J. G., Mungal, M. G., Kenny, T. W., Garguilo, M. G.: Electroosmotic Capillary Flow with Nonuniform Zeta Potential. Anal. Chem. 72, 10531057 (2000) 
[29] Ross, D., Johnson, T. J., Locascio, L. E.: Imaging of Electroosmotic Flow in Plastic Microchannels. Anal. Chem. 73, 2509-2515 (2001)

[30] Cuenca, A., Bodiguel, H.: Fluorescence photobleaching to evaluate flow velocity and hydrodynamic dispersion in nanoslits. Lab Chip 12, 1672-9 (2012)

[31] Brunet, F., Cid, E., Bartoli, A.: Simultaneous Image Registration and Monocular Volumetric Reconstruction of a Fluid Flow. Proc. Br. Machine vis. conf., 83.1-83.11 (2011)

[32] Braun, S.: The synchronous (time domain) average revisited. Mech. Syst. Sig. Process. 25, 1087-1102 (2011)

[33] Bair, S.: Pressure-Viscosity Behavior of Lubricants to 1.4 GPa and Its Relation to EHD Traction. Tribol. Trans. 43, 91-99 (2000)

[34] Fomina, N., McFearin, C., Sermsakdi, M., Edigin, O., Almutairi, A.: UV and near-IR triggered release from polymeric nanoparticles. JACS 132, 9540-2 (2010)

[35] Wang, G. R., Fiedler, H. E.: On high spatial resolution scalar measurement with LIF. Exp. Fluids 29, 265-274 (2000)

[36] Simon, J. R., Gough, a, Urbanik, E., Wang, F., Lanni, F., Ware, B. R., Taylor, D. L.: Analysis of rhodamine and fluorescein-labeled F-actin diffusion in vitro by fluorescence photobleaching recovery. Biophysical journal 54, 801-15 (1988)

[37] Jönsson, P., Jonsson, M. P., Tegenfeldt, J. O., Höök, F.: A method improving the accuracy of fluorescence recovery after photobleaching analysis. Biophys. J. 95, 5334-48 (2008)

[38] Sarkar, N., Das, K., Nath, D. N., Bhattacharyya, K.: Twisted charge transfer processes of nile red in homogeneous solutions and in faujasite zeolite. Langmuir 10, 326-329 (1994)

[39] Garbe, C.: Fluid flow estimation through integration of physical flow configurations. Pattern Recognit. LNCS 4713, 92-101 (2007)

[40] Mishra, D. K., Dolan, K. D., Yang, L.: Confidence intervals for modeling anthocyanin retention in grape pomace during nonisothermal heating. J. Food Sci. 73, E9-15 (2008)

[41] Reddyhoff, T., Spikes, H. a., Olver, a. V.: Compression Heating and Cooling in Elastohydrodynamic Contacts. Tribol. Lett. 36, 69-80 (2009)

[42] Fu, Z., Guo, F., Wong, P. L.: Friction-speed characteristics of elastohydrodynamically lubricated contacts with anomalous film shapes. Proc. Inst. Mech. Eng., Part J 226, 81-86 (2011)

[43] Boggs, J., Sibbitt, W.: Thermal Conductivity Measurements of Viscous Liquids. Ind. Eng. Chem. 47, 53-53 (1955)

[44] Guo, F., Wong, P. L.: An Anomalous Elastohydrodynamic Lubrication Film: Inlet Dimple. J. Tribol. 127, 425 (2005)

[45] Hartinger, M., Dumont, M.-L., loannides, S., Gosman, D., Spikes, H.: CFD Modeling of a Thermal and Shear-Thinning Elastohydrodynamic Line Contact. J. Tribol. 130, 041503 (2008) 
[46] Goyon, J., Colin, A., Bocquet, L.: How does a soft glassy material flow: finite size effects, non local rheology, and flow cooperativity. Soft Matter 6, 2668 (2010)

[47] Guo, Z., Zhao, T. S., Xu, C., Shi, Y.: Simulation of fluid flows in the nanometer: kinetic approach and molecular dynamic simulation. Int. J. Comp. Fluid Dyn. 20, 361-367 (2006)

[48] Fillot, N., Berro, H., Vergne, P.: From Continuous to Molecular Scale in Modelling Elastohydrodynamic Lubrication: Nanoscale Surface Slip Effects on Film Thickness and Friction. Tribol. Lett. , 257-266 (2011)

[49] Savio, D., Fillot, N., Vergne, P., Zaccheddu, M.: A Model for Wall Slip Prediction of Confined n-Alkanes: Effect of Wall-Fluid Interaction Versus Fluid Resistance. Tribol. Lett. 46, 11-22 (2012)

[50] Kaneta, M., Yang, P.: Effects of the thermal conductivity of contact materials on elastohydrodynamic lubrication characteristics. Proc. Inst. Mech. Eng., Part C 224, 25772587 (2010)

[51] Bruyere, V., Fillot, N., Morales-Espejel, G. E., Vergne, P.: Computational fluid dynamics and full elasticity model for sliding line thermal elastohydrodynamic contacts. Tribol. Int. 46, 3-13 (2012)

[52] Bair, S., Liu, Y., Wang, Q. J.: The Pressure-Viscosity Coefficient for Newtonian EHL Film Thickness With General Piezoviscous Response. J. Tribol. 128, 624 (2006)

[53] Hu, Y.-Z., Wang, H., Guo, Y., Shen, Z.-J., Zheng, L.-Q.: Simulation of lubricant rheology in thin film lubrication Part 2: simulation of Couette flow. Wear 196, 249-253 (1996)

[54] Mokshin, A. V., Barrat, J.-L.: Shear-induced crystallization of an amorphous system. Phys. Rev. E 77, 021505 (2008) 